\title{
Emergence of enterovirus 71 C4a in Denmark, 2009 to 2013
}

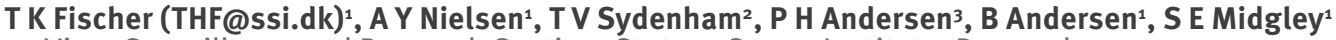

1. Virus Surveillance and Research Section, Statens Serum Institute, Denmark

2. Department of Clinical Microbiology, Odense University Hospital, Denmark

3. Department of Infectious Disease Epidemiology, Statens Serum Institute, Denmark

Citation style for this article:

Fischer TK, Nielsen AY, Sydenham TV, Andersen PH, Andersen B, Midgley SE. Emergence of enterovirus 71 C4a in Denmark, 2009 to 2013. Euro Surveill. 2014;19(38):pii=20911. Available online: http://www.eurosurveillance.org/ViewArticle. aspx?Articleld=20911

Article submitted on 26 February 2014 / published on 11 September 2014

Enterovirus (EV) 71 has emerged as a primary cause of severe neurologic enterovirus infection in the aftermath of the global polio eradication effort. Eleven subgenotypes of $E_{71}$ exist, the $C_{4}$ subgenotype being associated with large outbreaks in Asia with high mortality rates. This subgenotype has rarely been reported in Europe. In the period between 1 January 2009 and 31 December 2013 a total of 1,447 EV positive samples from 1,143 individuals were sent to the Statens Serum Institute (SSI), and 938 samples from 913 patients were genotyped at the Danish National World Health Organization Reference laboratory for Poliovirus at SSI. Echovirus 6 (Eo6) ( $n=141$ patients), echovirus 30 (E30) $(n=114)$, coxsackievirus A6 (CA06) $(n=96)$ and $\mathrm{EV}_{71}(\mathrm{n}=63)$ were the most prevalent genotypes. We observed a shift in circulating EV$_{71}$ subgenotypes during the study period, with subgenotype $\mathrm{C}_{4}$ dominating in 2012. A total of $34 \mathrm{EV}_{71}$ patients were found to be infected with strains of the $C_{4}$ subgenotype, and phylogenetic analysis revealed that they belonged to the $\mathrm{C}_{4} \mathrm{a}$ lineage. In our study, the proportions of cases with cerebral and/or sepsis-like symptoms were similar in those affected by $\mathrm{C}_{4} \mathrm{a}(19 / 34)$ and those with $\mathrm{C}_{1}$ and $C_{2}(15 / 35)$. The majority $(n=30)$ of the $34 E_{71} C_{4}$ cases were children $\leq 5$ years of age, and males $(n=22)$ were over-represented. Continued EV surveillance is required to monitor the spread of $\mathrm{EV}_{71} \mathrm{C}_{4}$ in Denmark and the rest of Europe.

\section{Introduction}

Human enteroviruses (EV) are small, single-stranded RNA-viruses from the Enterovirus genus of the Picornaviridae family. They can cause a range of clinical manifestations from mild mucocutaneous and/ or gastrointestinal symptoms, to visceral and severe neurologic diseases with involvement of central nervous system (CNS). Polioviruses used to be the most important EV due to widespread outbreaks of paralytic disease. A rather successful global effort to eradicate polio has now made $\mathrm{EV}_{71}$ the primary cause of severe neurotropic EV-associated infectious diseases [1]. EV71 variants have been classified into three genogroups ( $\mathrm{GgA}, \mathrm{GgB}$, and $\mathrm{GgC})$, and the latter two are further subdivided into subgenotypes $B_{1}$ to $B_{5}$, and $C_{1}$ to $C_{5}$. Currently genogroups $B$ and $C$ are co-circulating worldwide. Subgenotype $\mathrm{C}_{1}$ is predominating in Europe, but it can also be found in Australia, Malaysia and Singapore. The $\mathrm{C}_{4}$ subgenotype has predominantly been identified in large outbreaks of hand, foot and mouth disease (HFMD) in Asia, and in particular mainland China, where severe cases and a rather high mortality rate have been reported [2-4]. In 2004 the $\mathrm{C}_{4}$ subgenotype was detected for the first time in Europe, and has to date only been reported in a total of nine cases in Austria, Croatia, and Hungary, respectively [2-4]. In February 2012, the first EV71 C4 case was detected in Denmark in a Serbian infant admitted to the paediatric ward at Hospital A with fever and CNS symptoms. In the following months more $\mathrm{EV}_{71} \mathrm{C}_{4}$ cases were detected in the same geographical area as the hospital. The Virology Surveillance and Research Section (VOF) at the Department of Microbiological Diagnostics and Virology, Statens Serum Institut (SSI) serves as the National World Health Organization (WHO) Reference Laboratory for Poliovirus in Denmark. The Danish EV surveillance is implemented to monitor poliomyelitis as part of the polyomyelitis elimination efforts in Denmark. We took advantage of the wellfunctioning EV-surveillance system to characterise the emergence of $\mathrm{EV}_{71} \mathrm{C}_{4}$ strains in Denmark.

\section{Methods}

Enterovirus surveillance system

The national EV surveillance system in Denmark is conducted in a joint effort by the National WHO Poliovirus Reference Laboratory at VOF, SSI and the Infectious Disease Epidemiology Department (IDED) at SSI. The system is voluntary and all types of EV positive sample material may be submitted for characterisation. However, as part of the global poliovirus elimination programme, in case a patient is diagnosed with EV in the cerebrospinal fluid (CSF), it is highly requested that CSF and stool are forwarded to the Danish WHO Reference Laboratory for Poliovirus for virus characterisation including culture and viral protein (VP) 1 / 
VP2 sequence-based typing. Samples for diagnostic testing may also be sent directly to SSI from general practitioners and hospitals. Once weekly, VOF, SSI reports the new EV positive cases to the WHO Regional Office for Europe (EURO) in Copenhagen, as well as to the IDED. To ensure that relevant clinical information is archived in the national EV surveillance database, the IDED sends a letter with a standardised questionnaire regarding information on the clinical symptoms (including information on acute flaccid paralysis) and a reminder to send stool for virus characterisation directly to the doctors/departments in charge of the EV-positive patients. Completed questionnaires are returned and data entered in the database by the IDED, SSI. To ensure completeness of clinical data information for this paper, we have contacted relevant hospital departments and asked for relevant information on all EV71 cases where clinical information was missing.

\section{Enterovirus characterisation}

Isolates from all severe (i.e. with meningitis, encephalitis and sepsis-like illness) EV positive cases are routinely typed centrally at the VOF at SSI by sequencing part of the VP2 gene from the polymerase chain reaction (PCR) product obtained directly from the diagnostic sample, as VP2 sequencing has been demonstrated to be more sensitive than $\mathrm{VP}_{1}$ sequencing [5]. VP1 sequencing is performed in cases where $\mathrm{VP}_{2}$ typing is unsuccessful, or for specific typing analyses. Nontypeable virus isolates are cultivated in two cell-lines according to WHO guidelines [6] and then characterised by VP1 and VP2 sequencing. For this study, all samples that were positive for $\mathrm{EV}_{71}$ in diagnostic $P C R$ were tested by $\mathrm{VP}_{1} / \mathrm{VP}_{2}$ sequencing. $\mathrm{VP}_{1}$ typing and sequencing was applied to comply with international EV characterisation standards. The sample materials for $\mathrm{EV}_{71} \mathrm{C}_{4}$ positive patients are further described (results section).

RNA was extracted from $200 \mu \mathrm{l}$ of CSF using the QiaCube with the Qia AMP DNA Blood Mini Kit (QIAGEN Nordic, Copenhagen, Denmark), or from $200 \mu$ of other sample material (such as faeces, swabs, biopsies) using the MagNa pure LC robot with the total nucleic acids kit (Roche Diagnostics A/S, Hvidovre, Denmark). Diagnostic PCR for EV was conducted as described previously [7]. $5 \mu \mathrm{l}$ of the extraction was used as template for PCR amplifying part of the VP1 and VP2 gene, respectively in semi-nested PCR [5]. cDNA synthesis and first round PCR was carried out using a OneStep reverse transcription (RT)-PCR kit (QIAGEN Nordic, Copenhagen, Denmark), and second round amplification was carried out, producing a VP1 amplicon of 350 to 400 basepairs and a VP2 PCR amplicon of 368 basepairs.

Prior to sequencing, $P C R$ products were purified using exo-SAP IT (GE Healthcare, Buckinghamshire, UK). Purified PCR products were sequenced in both directions. Phylogenetic analysis based on the VP1

\section{FIGURE 1}

Distribution of enterovirus genotypes, Denmark, 2009-2013 (n=938) ${ }^{\mathrm{a}}$

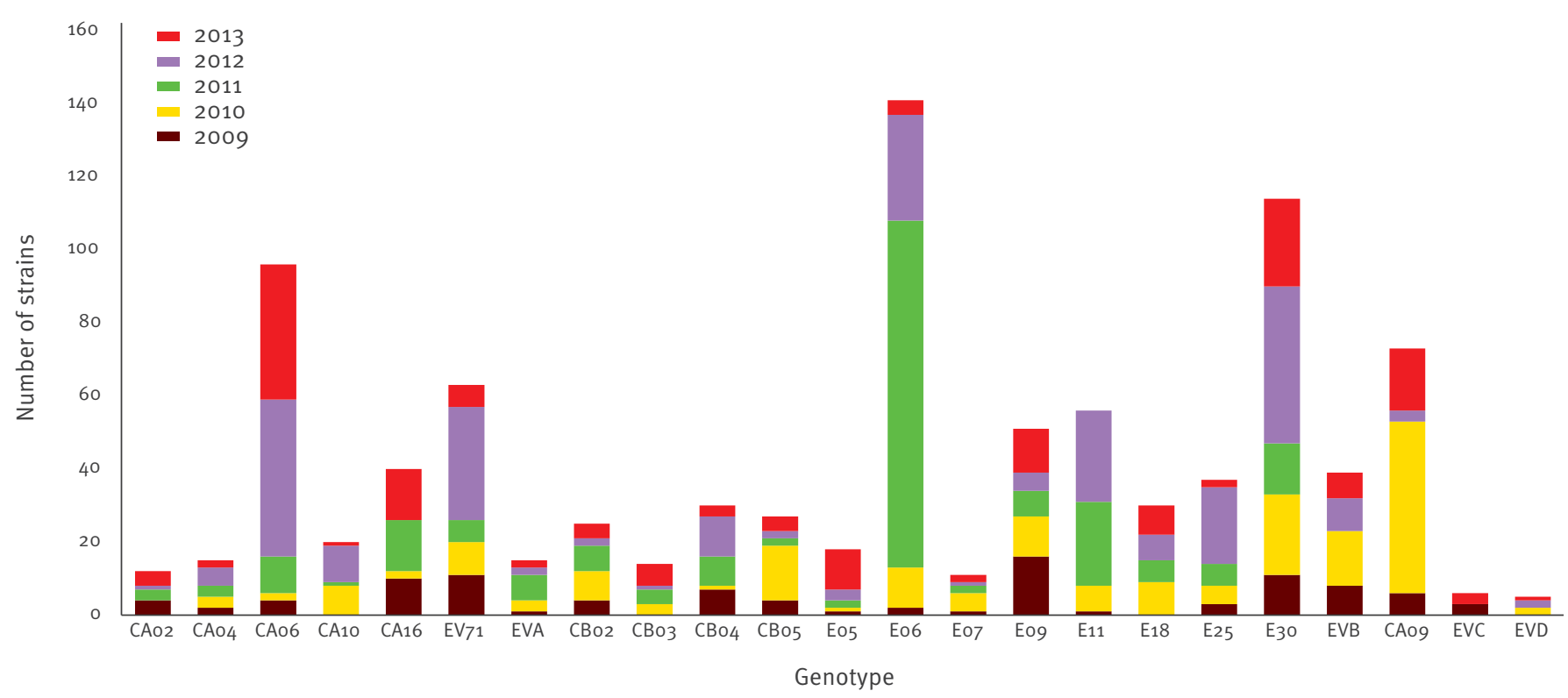

Genotypes for which 10 or more viruses were detected are shown. Genotypes for which fewer than 10 viruses were detected are grouped together according to which enterovirus (EV) species they belong to, i.e. EVA, EVB, EVC, and EVD. The viruses which are grouped according to species are shown immediately after the last genotype of that species in the chart.

a A total of 938 strains were characterised from 913 individuals. Of the 938 strains, 25 represent either co-infections with two different genotypes, or a subsequent infection with a different genotype. 


\section{FIGURE 2}

Distribution of enterovirus 71 subgenotypes C1, C2, and C4, Denmark, 2009-2013 ( $\mathrm{n}=63)$

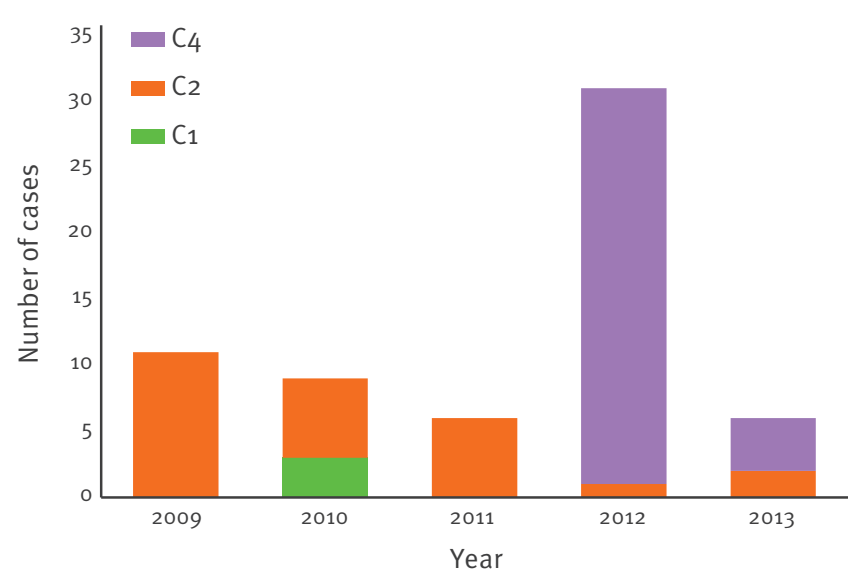

sequences was carried out by maximum likelihood and the Kimura 2-parameter model with discrete gamma distribution and invariable sites, and 1,000 bootstrap replications using the Molecular Evolutionary Genetics Analysis (MEGA) 5 software package [8]. VP1 sequence data from 23 of the 34 EV71 subgenotype $C_{4}$ samples were of sufficient quality and length to be included in the phylogenetic analysis (sequence length 178-305 nucleotides). All sequences from this study have been submitted to GenBank. Two subgenotype $C_{1}$ strains and 11 subgenotype $C_{2}$ strains were also included, as were all publically available (GenBank) European EV71 $\mathrm{C}_{4}$ sequences. The analysis was supplemented with reference sequences obtained from GenBank representing $E_{71}$ subgenotypes $A, B_{1}$ to $B_{5}$, and $C_{1}$ to $C_{5}$ (including $\mathrm{C}_{4} \mathrm{a}$ and $\mathrm{C}_{4} \mathrm{~b}$ ).

\section{Results}

In the period from 1 January 2009 to 31 December, 2013 a total of 7,879 samples from 5,611 individuals were received at the Department of Microbiology Diagnostic and Virology at SSI for EV diagnostics. Of these, 984 (12\%) samples from 779 (14\%) different individuals were positive for EV. In the same period 544 EV positive samples from 427 individuals were received for our national surveillance, thus totalling 1,447 positive samples from 1,143 individuals. 938 EV samples from 913 individuals were successfully genotyped. In 25 individuals more than one EV genotype was detected. A total of 41 different genotypes were identified, the most prevalent being Eo6 ( $n=141 / 913,15 \%)$, E30 $(n=114 / 913$, $12 \%)$, CAo6 ( $n=96 / 913,11 \%)$, and EV71 $(n=63 / 913,7 \%)$ (Figure 1).

$\mathrm{EV}_{71}$ was detected throughout the entire period, however there was a marked shift in the subgenotype, from $C_{1}(n=3)$ and $C_{2}(n=23)$, being found mainly between 2009 and 2011 with additionally $C_{2}$ in $2012(n=1)$ and $2013(n=2)$, to subgenotype $C_{4}$ being found primarily in $2012(n=30)$ but also in $2013(n=4)$ (Figure 2). The 34 $\mathrm{EV}_{71} \mathrm{C}_{4}$ infected individuals were unevenly distributed with regard to sex, as 22 of the 34 cases were males. With regards to age, the majority of infected individuals were young children, with 30 of the $34 \mathrm{C}_{4}$ cases $\leq 5$ years-old, and $16<1$ year-old.

With regard to the severity of symptoms, patients infected with the $\mathrm{C}_{4}$ subgenotype showed comparable symptoms to patients infected with subgenotypes $\mathrm{C}_{1}$ and/or C2 (Table 1 and 2). Nineteen of the $34 \mathrm{C}_{4}$ patients had cerebral or sepsis-like symptoms. Additional symptoms among the $\mathrm{EV}_{71} \mathrm{C}_{4}$ infected cases were gastroenteritis $(n=7)$, and HFMD $(n=4) . E_{71} C_{4}$ was detected primarily from stool samples $(n=19 / 34$, Table 1). Except for the single clustering of $\mathrm{EV}_{71} \mathrm{C}_{4}$ cases in Funen during the months of July to December of 2012 $(n=12)$, most single cases appear sporadically throughout the study period and from all five major geographical regions of Denmark (Table 1 ). Of the 12 clustered cases from Funen, 10 were admitted to the central hospital and one case was referred to this hospital from a nearby provincial hospital. The age range was o to 40 years (median: 2 years), with an uneven sex distribution of nine males, and three females.

The phylogenetic analysis revealed one major $\mathrm{C}_{4}$ lineage, containing all of the $\mathrm{C}_{4}$ strains reported in this study (Figure 3). These were determined to belong to the $\mathrm{C}_{4} \mathrm{a}$ lineage from Asia. Previously reported $\mathrm{C}_{4}$ strains from Europe belong to the $C_{4} b$ lineage [2-4].

\section{Discussion}

This study reports the finding of a new EV71 C $4 a$ subgenotype, detected in Denmark for the first time in the spring of 2012. As of December 2013 a further 33 EV71 $\mathrm{C}_{4}$ cases have been detected, the majority in infants with moderate to severe symptoms. $\mathrm{EV}_{71} \mathrm{C}_{4}$ cases from Austria and Hungary were also found to be associated with severe symptoms such as meningitis and acute flaccid paralysis $[2,3]$. In Denmark, study material is based on cases referred for either diagnostic purposes, or submitted to the National WHO Polio Reference Laboratory at SSI, as part of the national EV surveillance. As a consequence, the detection of mild and/ or asymptomatic cases of EV71 infection in the Danish population is not complete, and we can therefore not conclude that $\mathrm{EV}_{71} \mathrm{C}_{4}$ is always associated with severe symptomatology. Only 6/63 EV71 cases were associated with HFMD. There is no specific surveillance for HFMD in Denmark, so the actual level of mild cases of $\mathrm{EV}_{71}$ in circulation may be underestimated.

The EV71 $\mathrm{C}_{4}$ strains identified in Denmark shared a surprisingly high sequence similarity with an $\mathrm{EV}_{71} \mathrm{C}_{4 \mathrm{a}}$ epidemic strain from China, 2008 (EU913466, Figure 3) $[9-11]$. So far, the relatively severe presentation, although with no fatalities, of 19 of the $34 \mathrm{EV}_{71} \mathrm{C}_{4}$ cases, with a temporal-spatial clustering of nine of the meningitis/encephalitis cases in Funen during the second half of 2012, suggests that the Danish emerging $\mathrm{C}_{4}$ strain has the same potential for a high transmission 
TABLE 1

Characteristics of enterovirus 71 C4 infected patients, Denmark, 2009-2013 ( $\mathrm{n}=34$ patients)

\begin{tabular}{|c|c|c|c|c|c|c|}
\hline $\begin{array}{l}\text { Patient } \\
\text { number }\end{array}$ & Age in Years & Sex & Date of sampling & $\begin{array}{l}\text { Geographical } \\
\text { region }\end{array}$ & $\begin{array}{c}\text { Primary } \\
\text { symptoms }\end{array}$ & Sample material \\
\hline 1 & 0 & $M$ & $11-02-2012$ & Funen & Meningitis & Faecal swab \\
\hline 2 & 0 & $M$ & $22-05-2012$ & Jutland & Meningitis & Stool \\
\hline 3 & 2 & $M$ & $06-07-2012$ & Funen & Gastroenteritis & Swab \\
\hline 4 & 4 & M & $09-07-2012$ & Funen & Meningitis & Faecal swab \\
\hline 5 & 3 & $\mathrm{~F}$ & $23-07-2012$ & Jutland & Encephalitis & Stool \\
\hline 6 & 0 & $\mathrm{~F}$ & $01-08-2012$ & Jutland & Gastroenteritis & Stool \\
\hline 7 & 0 & $\mathrm{~F}$ & $22-08-2012$ & Funen & Sepsis-like syndrome & Stool \\
\hline 8 & 40 & $M$ & 30-08-2012 & Funen & Meningitis & CSF \\
\hline 9 & 2 & $M$ & $04-09-2012$ & Funen & Meningitis & Stool \\
\hline 10 & 0 & $\mathrm{~F}$ & $11-09-2012$ & Zealand & Fever & Stool \\
\hline 11 & 1 & $M$ & $13-09-2012$ & Zealand & Unknown & Fluid \\
\hline 12 & 6 & $M$ & 27-09-2012 & Jutland & HFMD & Swab \\
\hline 13 & 29 & M & $11-10-2012$ & Jutland & Respiratory symptoms & Swab \\
\hline 14 & 0 & $\mathrm{~F}$ & $14-10-2012$ & Jutland & Gastroenteritis & Stool \\
\hline 15 & 0 & M & $17-10-2012$ & Funen & Encephalitis & Swab \\
\hline 16 & 3 & M & $26-10-2012$ & Funen & Meningitis & Swab \\
\hline 17 & 0 & M & $26-10-2012$ & Jutland & Meningitis & Stool \\
\hline 18 & 1 & M & $30-10-2012$ & Funen & Encephalitis & Swab \\
\hline 19 & 0 & $M$ & $04-11-2012$ & Funen & Gastroenteritis & Stool \\
\hline 20 & 0 & M & $05-11-2012$ & Funen & Meningitis & Stool \\
\hline 21 & 0 & M & $07-11-2012$ & Zealand & Sepsis-like syndrome & Stool \\
\hline 22 & 0 & $\mathrm{~F}$ & $08-11-2012$ & Zealand & Sepsis-like syndrome & Stool \\
\hline 23 & 0 & $M$ & $09-11-2012$ & Funen & Meningitis & Stool \\
\hline 24 & 4 & M & $16-11-2012$ & Jutland & Meningitis & Stool \\
\hline 25 & 1 & $\mathrm{~F}$ & $25-11-2012$ & Jutland & Fever & Stool \\
\hline 26 & 1 & $\mathrm{~F}$ & 28-11-2012 & Jutland & Gastroenteritis & Stool \\
\hline 27 & 33 & $\mathrm{~F}$ & $06-12-2012$ & Zealand & HFMD & Vesicle fluid \\
\hline 28 & 2 & M & $07-12-2012$ & Zealand & HFMD & Swab \\
\hline 29 & 3 & $M$ & $11-12-2012$ & Jutland & Gastroenteritis & Stool \\
\hline 30 & 4 & $\mathrm{~F}$ & $18-12-2012$ & Funen & Meningitis & Swab \\
\hline 31 & 0 & M & $08-01-2013$ & Jutland & Gastroenteritis & Stool \\
\hline 32 & 2 & $\mathrm{~F}$ & $16-07-2013$ & Jutland & Meningitis+HFMD & Faecal swab \\
\hline 33 & 0 & M & $22-08-2013$ & Zealand & Meningitis & CSF \\
\hline 34 & 0 & $\mathrm{~F}$ & $03-10-2013$ & Jutland & Fever & Stool \\
\hline
\end{tabular}

CSF: cerebrospinal fluid; HFMD: hand foot and mouth disease; M: male; F: female.

rate and high pathogenicity as described previously for Asian $\mathrm{EV}_{71} \mathrm{C}$ strains, including $\mathrm{EV}_{71} \mathrm{C}_{4}$ [9-13].

Other $\mathrm{EV}_{71} \mathrm{C}$ subgenotypes, namely $\mathrm{C}_{1}$ and $\mathrm{C}_{2}$, have previously been identified as occurring sporadically in Denmark throughout a four year study period (2005 to 2008), implying simultaneous circulation of these lineages without genetic selection of either strain based on $\mathrm{VP}_{2}$ sequences [14]. However, only one $\mathrm{EV}_{71} \mathrm{C}_{2}$ case was identified during 2012 and two in 2013, suggesting that the introduction of $\mathrm{C}_{4}$ within the population of Denmark might have a suppressive impact on the circulation of other $\mathrm{EV}_{71} \mathrm{C}$ subgenotypes. Furthermore, the number of $E V_{71}$ positive samples in $2012(n=31)$ is in itself notable, as a total of only $29 \mathrm{EV}_{71}$ cases were identified throughout the previous four-year study period [11]. It will be interesting to follow the emergence of $\mathrm{C}_{4}$, and see whether it will follow the typical trend of the other $\mathrm{EV}_{71}$ subgenotypes with only limited evolutionary change within its two lineages ( $\mathrm{C}_{4} \mathrm{a}$ and $\mathrm{C}_{4} \mathrm{~b}$ ) over time, or whether this subgenotype will continue to dominate the future $\mathrm{EV}$ seasons and give rise to outbreaks of severe disease in Europe, as the $\mathrm{C}_{4} \mathrm{~S}$ are known for in parts of Asia. The increasing number of $\mathrm{EV}_{71} \mathrm{C}_{4}$ identified during the 2009 to 2013 surveillance period, and the initial clustering of 11 cases within one 
TABLE 2

Main clinical presentation of enterovirus (EV)71 C4 and EV71 C1 and EV71 C2 patients, Denmark, 2009-2013 $(n=63)$

\begin{tabular}{|l|c|c|}
\hline Symptom & C $_{4}$ patients & C $_{2}+C_{1}$ patients \\
\hline Meningitis & 13 & 12 \\
\hline Gastroenteritis & 7 & 7 \\
\hline HFMD & 4 & 2 \\
\hline Encephalitis & 3 & 2 \\
\hline Sepsis-like syndrome & 3 & 1 \\
\hline Respiratory symptoms & 1 & 1 \\
\hline Fever & 3 & 6 \\
\hline Myoclonus & 0 & 1 \\
\hline Unknown & 1 & 1 \\
\hline Total number of patients & $34^{\mathrm{a}}$ & $29^{\mathrm{a}}$ \\
\hline
\end{tabular}

HFMD: hand foot and mouth disease.

Some patients presented more than one symptom so the total numbers of patients are not equal to the sum of the numbers in the respective columns.

hospital during the second half of 2012, suggest that this subgenotype initially gave rise to a smaller localised outbreak and is potentially now establishing itself in this northern European population. In the recent 'perspective' from the Global Disease Detection Operations Center at the United States Centers for Disease Control and Prevention (CDC), EV71 was considered to be one of the top-five global infectious disease threats to watch, due to its propensity to cause large outbreaks and severe, life threatening, neurologic disease [15].

In conclusion, the circulation of EV71 subgenotype $\mathrm{C}_{4 \mathrm{a}}$ in Denmark has been established. Based on observations using a wide range of different samples from patients with a broad range of EV symptoms, this subgenotype was found to coincide with severe disease, as were the other EV71 subgenotypes $C_{1}$ and C2, detected in Denmark during the study period. EV surveillance of high quality and high sample volume is needed to closely monitor the continued emergence of $\mathrm{EV}_{71} \mathrm{C}_{4}$ in the European population over the coming years to establish the pathogenicity and virulence of this subgenotype. A broader emergence of $E_{71}$ within Europe might potentially widen the focus of the current development of EV71 vaccines for targeted use in Asia, to a potential future benefit in Europe as well $[16,17]$.

\section{FIGURE 3}

Phylogenetic analysis of viral protein 1 sequences from Danish enterovirus 71 strains, Denmark, 2009-2013

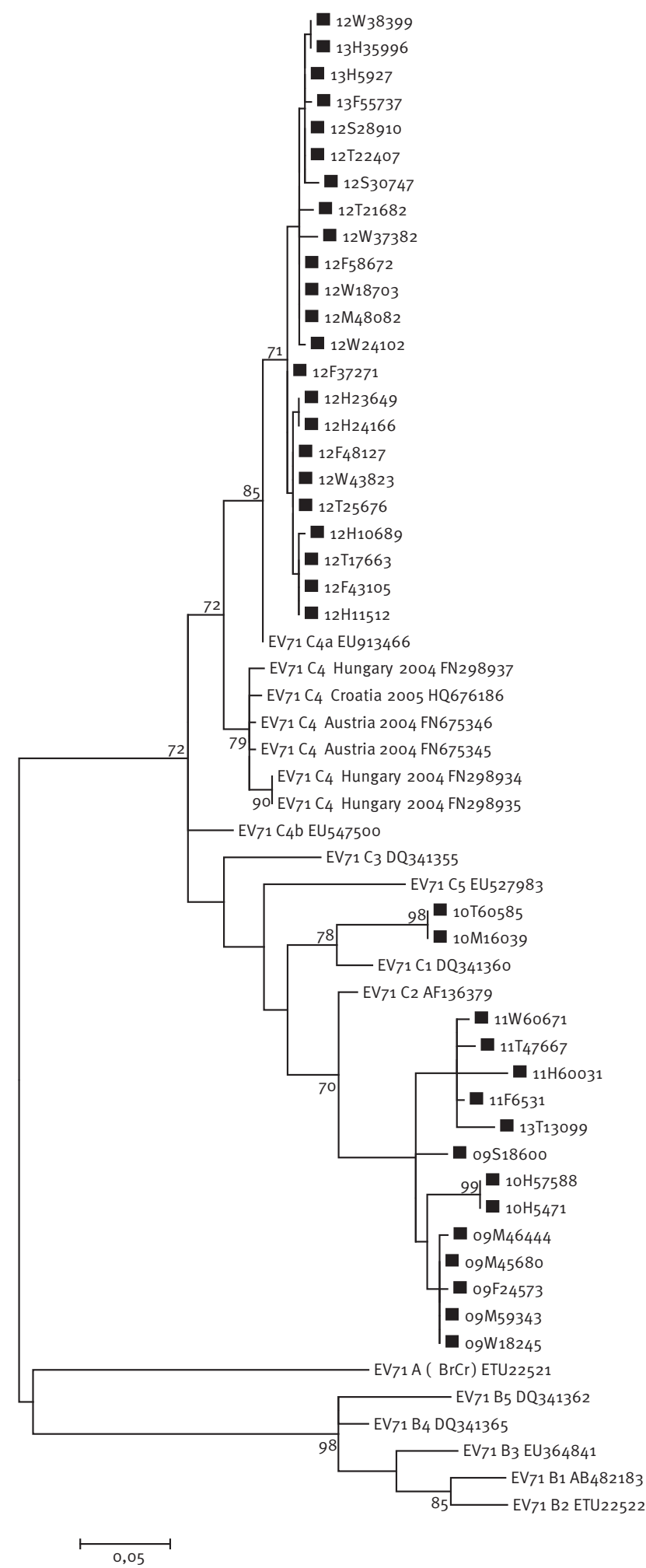

Danish enterovirus (EV)71 strains are represented with a black square. The sequence identifiers for the sequences obtained in this study are made up of a two figure prefix to denote the year of sample collection, followed by our internal sample number. Subgenotype $C$ reference sequences were downloaded from GenBank (EV71 $\mathrm{C}_{1}$ accession number: DQ341360; EV71 C2: AF136379; EV71 C3: DQ341355; EV71 C4a: EU913466; EV71 C4b: EU 547500; EV71 C5: EU 527983). EV71 subgenotypes A (EV71A: ETU22521) and B (B1: AB482183; B2: ETU22522; B3: EU364841; $\mathrm{B}_{4}$ : DQ341365; B5: DQ341362) were included to root the tree. Previously identified European $\mathrm{C}_{4}$ strains were also included in the analysis. The scale bar represents the number of nucleotide substitutions per site. 


\section{Acknowledgements}

We are grateful to all the Regional Danish Microbiological Departments for submission of all enterovirus positive samples to the Virus Surveillance and Research laboratory at Statens Serum Institute. Further we thank Mrs AS Hintzmann for her contribution to the enterovirus surveillance during 2013 and Mrs M Jorgensen for her assistance with grammatical review of the manuscript.

\section{Conflict of interest}

None declared.

\section{Authors' contributions}

TKF: conceptualised the study, drafted the manuscript and headed the investigation; AYN: participated in the molecular and phylogentic analyses, and has participated in the writing of the manuscript; TVS: collected clinical information on the most important samples; PHA: participated in the national enterovirus surveillance and in the registration of clinical symptoms; BA: conducted the laboratory characterisation of $\mathrm{EV}$; SOI: headed the laboratory characterisation of EV and the phylogenetic analyses, and has participated in the writing of the manuscript.
12. Chua KB, Kasri AR. Hand foot and mouth disease due to enterovirus 71 in Malaysia. Virol Sin. 2011;26(4):221-8. http:// dx.doi.org/10.1007/s12250-011-3195-8

13. Liu Y, Fu C, Wu S, Chen X, Shi Y, Zhou B, et al. A novel finding for enterovirus virulence from the capsid protein $V_{P_{1}}$ of $E V_{71}$ circulating in mainland China. Virus Genes. 2014;48(2):260-72. http://dx.doi.org/10.1007/s11262-014-1035-2

14. Badran SA, Midgley S, Andersen P, Böttiger B. Clinical and virological features of enterovirus 71 infections in Denmark, 2005 to 2008 . Scand J Infect Dis. 2011;43(8):642-8. http:// dx.doi.org/10.3109/00365548.2011.577094

15. Christian KA, Ijaz K, Dowell SF, Chow CC, Chitale RA, Bresee JS, et al. What we are watching--five top global infectious disease threats, 2012: a perspective from CDC's Global Disease Detection Operations Center. Emerg Health Threats J. 2013;6:20632.

16. Pallansch MA, Oberste MS. Enterovirus 71 encephalitis: a new vaccine on the horizon?. Lancet. 2013;381(9871):976-7. http:// dx.doi.org/10.1016/S0140-6736(13)60286-X

17. Zhu FC, Liang ZL, Li XL, Ge HM, Meng FY, Mao OY, et al. Immunogenicity and safety of an enterovirus 71 vaccine in healthy Chinese children and infants: a randomised, double-blind, placebo-controlled phase 2 clinical trial. Lancet. 2013;381(9871):1037-45. http://dx.doi.org/10.1016/ So140-6736(12)61764-4

\section{References}

1. Qiu J. Enterovirus 71 infection: a new threat to global public health? Lancet Neurol. 2008;7(10):868-9. http://dx.doi. org/10.1016/S1474-4422(08)70207-2

2. Huemer HP, Ortner B, Huang CW, Schmid D, Mutz I, Wewalka $\mathrm{G}$, et al. Isolating Asian enterovirus 71 subgenogroup $C_{4}$ in two Austrian clinical samples from 2004. Euro Surveill. 2008;13(28): $\mathrm{pii}=18922$.

3. Kapusinszky B, Szomor KN, Farkas A, Takács M, Berencsi G. Detection of non-polio enteroviruses in Hungary 2000-2008 and molecular epidemiology of enterovirus 71, coxsackievirus A16, and echovirus 30. Virus Genes 2010;40(2):163-73. http:// dx.doi.org/10.1007/s11262-009-0440-4

4. Mirand A, Schuffenecker I, Henquell C, Billaud G, Jugie G, Falcon D, et al. Phylogenetic evidence for a recent spread of two populations of human enterovirus 71 in European countries. J Gen Virol. 2010;91(Pt 9):2263-77. http://dx.doi. org/10.1099/vir.0.021741-0

5. Nasri D, Bouslama L, Omar S, Saoudin H, Bourlet T, Aouni M, et al. Typing of human enterovirus by partial sequencing of VP2. J Clin Microbiol. 2007;45(8):2370-9. http://dx.doi.org/10.1128/ JCM.00093-07

6. Vaccines and Biologicals, World Health Organization. Guidelines for environmental surveillance of poliovirus circulation. Geneva:WHO;2003.

7. Nielsen AC, Böttiger B, Midgley SE, Nielsen LP. A nove enterovirus and parechovirus multiplex one-step realtime PCR-validation and clinical experience. J Virol Methods. 2013;193(2):359-63. http://dx.doi.org/10.1016/j. jviromet.2013.06.038

8. Tamura K, Peterson D, Peterson N, Stecher G, Nei M, Kumar S. MEGA5: molecular evolutionary genetics analysis using maximum likelihood, evolutionary distance, and maximum parsimony methods. Mol Biol Evol. 2011;28(10):2731-9. http:// dx.doi.org/10.1093/molbev/msr121

9. Ho M, Chen ER, Hsu KH, Twu SJ, Chen KT, Tsai SF, et al. An epidemic of enterovirus 71 infection in Taiwan. $N$ Eng J Med. 1999;341(13):929-35. http://dx.doi.org/10.1056/ NEJM199909233411301

10. Wang LC, Tang SQ, Li YM, Zhao HL, Dong CH, Cui PF, et al. A comparison of the biological characteristics of $\mathrm{EV}_{71} \mathrm{C}_{4}$ subtypes from different epidemic strains. Virol Sin. 2010;25(2):98-106. http://dx.doi.org/10.1007/ S12250-010-3102-8

11. Zhang Y, Tan X, Cui A, Mao N, Xu S, Zhu Z, et al. Complete genome analysis of the $\mathrm{C}_{4}$ subgenotype strains of enterovirus 71: predominant recombination $\mathrm{C}_{4}$ viruses persistently circulating in China for 14 years. PLoS One. 2013;8(2):e56341. http://dx.doi.org/10.1371/journal.pone.0056341 